\title{
Configuraciones tonales de frases entonativas en enunciados aseverativos del español de la Ciudad de México
}

\author{
Intonational Configurations of Intonational Phrases in \\ Declarative Statements from the Spanish Variety \\ of Mexico City
}

\author{
Alberto Rodríguez Márquez \\ Universidad Nacional Autónoma de México \\ Alberto.mrm@gmail.com
}

Resumen

El objetivo de este trabajo es describir prosódicamente las configuraciones tonales finales de las frases entonativas intermedias (ip) y los tonemas de las frases entonativas mayores (IP) en el habla de la Ciudad de México. Para ello se empleó un corpus de habla espontánea conformado por hablantes de dos redes sociales: red vecinal y red laboral. Las configuraciones tonales finales de ip presentan una inflexión principalmente ascendente con una mayor duración en la sílaba linde. La diferencia más significativa entre ambas redes sociales radica en la duración de las configuraciones finales oxítonas, más prolongada en la red laboral. En los tonemas se registra un mayor alargamiento por parte de la red vecinal y un predominio general de descensos tonales, aunque el tonema circunflejo representa un número importante de los casos.

Palabras Clave: prosodia, entonación, frase entonativa intermedia, frase entonativa mayor, redes sociales, prosodia basada en el uso, prosodia enunciativa, español de México

\section{Abstract}

The objective of this paper is to describe the prosodic features of the final intonation contour of minor intonational phrases (ip) and the tonemes of major intonational phrases (IP) in Mexico City's Spanish variety. The speech data was taken from a spontaneous speech corpus made from speakers from two social networks: neighborhood and labor. Final intonation contours of ip show a predominantly rising movement. These contours are generally produced with greater length in the last syllable of the ip, which represents the most significant difference between both networks in the case of oxitone endings. On the other hand, tonemes are predominantly descendant, although the circumflex accent has an important number of cases within the data set. Tonemes produced by the neighborhood network are produced with larger length than those from the labor network. 
KEYwORDS: prosody, intonation, minor intonational phrase, major intonational phrase, social networks, usage-based prosody, propositional prosody, Mexican Spanish

FECHA DE RECEPCIÓN: 28/02/2020

FECHA DE ACEPTACIÓN: 03/07/2020

\section{Introducción}

Actualmente, existe una multiplicidad de perspectivas para el estudio de los fenómenos prosódicos en las diferentes lenguas. En el caso concreto de esta investigación, se emplean dos perspectivas de análisis: el de la prosodia basada en el uso (PBU) y el de la prosodia enunciativa. El primero de ellos, estrechamente relacionado con la gramática basada en el uso (Bybee, 2001), busca brindar una perspectiva realista de los procesos lingüísticos al enfocarse, entre otros aspectos, en que la frecuencia con la que se da una estructura permite observar su asentamiento social, la negociación de identidades de los hablantes, el aprovechamiento conversacional y el aprendizaje (Martín Butragueño, 2015).

Por su parte, la prosodia enunciativa "estudia todos los fenómenos prosódicos que se encuentran por encima del nivel de la palabra fonológica o palabra prosódica y que tienen una utilidad lingüística cuando menos comunicativa" (Martín Butragueño, 2019, p. 19). Para obtener una mejor comprensión de la relación que guarda este concepto con los niveles prosódicos que se tratan en este trabajo (frase entonativa intermedia y mayor), resulta conveniente explicar tanto la relación de dichos niveles en la jerarquía prosódica como su utilidad comunicativa.

Las representaciones fonológicas de los elementos suprasegmentales, de acuerdo con Selkirk (1986), consisten en una serie de constituyentes prosódicos o categorías que se encuentran jerarquizados de manera que cada nivel puede servir como dominio para la aplicación de ciertas reglas fonológicas específicas. Esta autora también señala que dicha jerarquía no tiene una relación isomórfica con la estructura sintáctica, pero se define en relación con ella, al menos par- 
cialmente. Hasta el momento, se han identificado siete niveles dentro de la jerarquía prosódica (D’Introno, Del Teso y Weston, 2010; Nespor y Voguel, 2007; Selkirk 1986), los cuales se presentan a continuación iniciando desde el nivel más alto y concluyendo con el más bajo: enunciado fonológico $(U)$, frase entonativa $(I)$, frase fonológica $(\phi)$, grupo clítico $(C)$, palabra fonológica $(\omega)$, pie silábico $(\Sigma)$ y sílaba $(\sigma)$.

Si bien este modelo de jerarquía parece ser el más extendido, se han presentado algunas variantes. Tales son los casos de Prieto (2006) y Toledo (2008a). La primera autora presenta la siguiente jerarquía consistente en cinco niveles: IP, frase entonativa (también llamada frase mayor); $\mathrm{PPh} o \varphi$, frase fonológica (también llamada frase menor); PW o $m$, palabra prosódica; F, pie silábico; $\sigma$, sílaba. Toledo (2008a), cuyo planteamiento se emplea como guía para la designación de los niveles estudiados en este trabajo, propone la siguiente jerarquía prosódica que, aunque es bastante similar a la anterior, contiene algunos cambios en cuanto a nomenclaturas y número de niveles: frase entonativa mayor (IP), frase entonativa intermedia (ip), frase fonológica $(\phi)$, palabra prosódica (u) y sílabas $(\sigma)$.

Es pertinente recalcar que, tal como se mencionó anteriormente, en esta investigación solo se tomarán en cuenta los dos niveles más altos de la jerarquía prosódica (frase entonativa mayor y frase entonativa intermedia). Por lo que el debate acerca de la adecuación o validez de los demás niveles jerárquicos en el estudio prosódico del español queda fuera de los límites de este trabajo. Adicionalmente, también vale la pena señalar que se emplean los términos frase entonativa mayor (IP) y frase entonativa intermedia (ip) para hacer referencia, respectivamente, a enunciado fonológico $(U)$ y frase entonativa (I). Esta decisión no representa un conflicto con ninguno de los modelos jerárquicos presentados, pues, en todo caso, estos elementos se corresponden con los dos niveles más altos de la jerarquía prosódica (los cuales siempre son superiores a la frase fonológica).

Aunque la idea de que los niveles jerárquicos previamente descritos es ampliamente aceptada entre los lingüistas, algunos 
consideran que estos niveles no aplican a todas las lenguas. Sosa afirma que, a pesar de que el planteamiento de distintos tipos de grupos melódicos ha sido identificado productivamente en ciertas lenguas (como el inglés y el japonés), en el caso del español "no encontramos justificación alguna para postular más de un tipo de grupo melódico, por lo que entendemos que hay un solo nivel o dominio, definido por el contorno entonacional y delimitado por el tonema" (1999, pp. 55-56). No obstante, investigaciones como las de Prieto (2006), Toledo (2007, 2008a, 2008b) y Martín Butragueño (2019) han empleado distintos niveles inferiores a la frase entonativa mayor (IP) o enunciado fonológico $(U)$ en sus trabajos, lo que no solo da cuenta de la existencia de distintos niveles en el fraseo fonológico, sino de la productividad y utilidad lingüística de estos elementos en la lengua española.

De acuerdo con von Heusinger (2007), las características que se le han otorgado a las IP no son exactamente concisas. No obstante, explica este autor, hay criterios fonéticos, sintácticos y semánticos que ayudan a delimitar una frase entonativa mayor: 1) puede estar precedido o seguido por una pausa;2) contiene la presencia del acento más prominente; 3 ) algunas veces, el linde está marcado por un tono de juntura; 4) el linde puede bloquear algunos fenómenos de juntura; 5) los lindes corresponden con los de algunos constituyentes sintácticos; 6) el material lingüístico debe constituir una unidad de sentido o información.

Las frases entonativas intermedias, en cambio, representan un grado menor de separación que el de las mayores (IP) (Hualde, 2003). Toledo (2007) menciona que las ip tienen las siguientes características: 1) poseen un acento prominente en la última sílaba tónica; 2) suele producirse entre pausas; 3 ) tienen, opcionalmente, un ascenso indicador de continuación en el linde derecho de la frase, aunque también es posible que la frase culmine sin que suceda dicho ascenso; 4) las ip que culminan con tonos altos ( $\left.\mathrm{H}^{-}\right)$presentan un crecimiento tonal en la última sílaba de la palabra fonológica final que es prolongado por el alargamiento temporal final producido en dicha sílaba; 5) cuando la ip culminan con tono bajo (L-) indican la separación de esa frase de la siguiente, mientras que aquellas que culminan 
con tono alto (H-) señalan que la frase tiene cohesión semántica con la siguiente frase. Este empleo de tonos $\mathrm{H}$ - para designar continuación es descrito como un hecho biológico por Gussenhoven (2004), quien lo menciona en su explicación del código de producción como un recurso empleado por los hablantes para señalar la no conclusión del enunciado en oposición a la declinación tonal, asociada con finalización de la enunciación, esperada por la pérdida de energía que se da desde el inicio de la producción oral. El uso de este recurso para indicar continuidad o no conclusión de la enunciación ha sido documentado en el español desde una perspectiva tanto fonológica (Navarro Tomás, 1957 y 1966) como fonética (Quilis, 1981).

Ahora bien, "el conjunto de tonos que marcan el final de un enunciado y que coincide con las sílabas finales a partir de la que lleva el último acento" recibe el nombre de tonema o núcleo (Sosa, 1999, p. 31). Si bien, en principio, este término puede aplicarse para hacer referencia al material lingüístico posterior a la última sílaba acentuada tanto en ip como en IP, se decidió mantener dicho término únicamente en lo referente a las IP. En este trabajo, al material prosódico dado desde la última sílaba acentuada de las ip hasta el final de estas se le llamará configuración tonal final de ip. Esto es porque se considera pertinente establecer una diferencia terminológica concreta entre los constituyentes tonales de ip e IP que obedezca a la diferencia jerárquica de ambos niveles prosódicos. Mientras que el tonema da un sentido de terminación a una unidad de sentido o información (una IP), incluso en aquellos casos en los que tal vez no exista una pausa que haga evidente su finalización, la configuración tonal final de ip no posee dicha característica. En este caso, la conclusión de una ip no implica la culminación de la emisión de una unidad completa de sentido, sino de una parte de ella, lo cual, como se explicó previamente, puede ser marcado a través de una juntura alta (H-) o una juntura baja (L-).

Las características de los tonemas en el español general han sido descritas previamente desde los trabajos de Navarro Tomás (1957 y 1966). En el caso del español de México, en estudios como los de Matluck (1952), Kvavik (1979) y Sosa (1999), entre 
otros, se ha señalado como característica del español del centro de México la producción de una cadencia circunfleja, relacionada con el tonema circunflejo $\mathrm{L}+\mathrm{H}^{*} \mathrm{~L} \%$. Adicionalmente, investigaciones como la de Martín Butragueño (2006) han ahondado en las variaciones sociolingüísticas de los tonemas en el habla de la Ciudad de México. No obstante, no ocurre lo mismo con la configuración tonal final de ip, acerca de la cual no se pudo encontrar mucha información en el habla de esta región. Los trabajos que se pudieron revisar contienen datos relacionados con el español peninsular (Toledo, 2007) y colombiano (Toledo, 2008b). En ambos casos, el acento nuclear de ip suele ser ascendente $\left(\mathrm{L}+\mathrm{H}^{*}\right)$ que continúa con un tono de juntura alto $(\mathrm{H}-)$ como señal de no continuidad y que contrasta con la juntura final baja (L\%) de IP, que designa conclusión.

Debido a esta falta de información acerca de las características de las configuraciones tonales finales de ip en el habla de México, este trabajo se propone como objetivo llevar a cabo una descripción de las características prosódicas, específicamente en término de duración y entonación, tanto de las configuraciones tonales finales de las de frases entonativas intermedias (ip) como de los tonemas de las frases entonativas mayores (IP) en el habla de la Ciudad de México. Adicionalmente, dado que las características del corpus lo permiten, se hará un contraste entre los rasgos prosódicos mencionados de dos redes sociales de esta ciudad. Esto no responde a la realización de un análisis sociolingüístico en profundidad, sino a la presentación de un panorama suficientemente amplio que permita observar y describir los rasgos prosódicos de las secciones finales de ambos tipos de frases en el habla de dicha ciudad.

\section{Metodología}

Los datos para llevar a cabo esta investigación consisten en muestras no controladas que fueron recolectadas a través de una entrevista sociolingüística guiada, mas no condicionada, por un repertorio de preguntas relacionadas con diferentes experiencias de vida de los entrevistados. Debido a que la prioridad era 
obtener datos lingüísticos producidos con la mayor naturalidad posible (dentro de las limitaciones contextuales de una entrevista), se permitió que los sujetos guiaran la conversación en la mayor medida de lo posible, mientras que el entrevistador intervenía lo menos posible, manteniendo actitud de aprendiz (Labov, 1981).

Los doce informantes que conforman la muestra se distribuyen equitativamente en dos redes sociales. La primera de ellas, denominada red social vecinal, está constituida por personas de colonias humildes adyacentes de la zona noroeste de la delegación Miguel Hidalgo de la Ciudad de México, quienes comparten vínculos vecinales, familiares y de amistad. Ninguno de los individuos tiene estudios universitarios; uno de ellos solo terminó la primaria, cuatro terminaron la secundaria y solo uno finalizó la preparatoria. En el ámbito laboral, de los seis informantes, cuatro son autoempleados (tres comerciantes ambulantes y un taxista), uno trabaja como personal de atención al público en una cafetería y el último coordina actividades culturales en su comunidad (trabaja en un centro comunitario). En términos financieros, el nivel de ingresos general se encuentra entre 2,700 y 5,400 pesos mensuales (de uno a dos salarios mínimos), por lo que se puede decir que se encuentran en un nivel socioeconómico bajo. Esto, aunado a algunas características que se pudieron observar y documentar, permite ubicar a los miembros de esta red dentro de los modos de vida B, D, E y F mencionados por Lastra y Martín Butragueño (2000).

La segunda red social, denominada red social laboral, está conformada por empleados con cargos ejecutivos de una empresa multinacional cuya sede central en México se encuentra en la colonia Polanco $1^{a}$ Sección (ubicada a unos dos kilómetros en línea recta de las colonias donde habitan los miembros de la red social vecinal). Los integrantes de esta red comparten lazos laborales y afectivos dentro del entorno laboral, pues todos ellos se conocen entre sí. En el aspecto educativo, todos los integrantes de esta red tienen títulos universitarios y la mayoría de ellos cuenta con posgrados. A pesar de que la mayoría de los integrantes de la red posee cargos con responsabilidades de gerencia y dirección, todos ellos comparten el hecho de que tienen superiores inmedia- 
tos que se encuentran en la misma oficina y de que tienen fuertes motivaciones de superación laboral. Por otra parte, todos los integrantes de esta red tienen un nivel de ingresos mucho más elevado, pues sus ingresos mensuales representan, cuando menos, más de cinco veces el ingreso mensual más elevado de la red vecinal. Todas estas características sitúan a los miembros de esta red en el modo de vida $\mathrm{G}$ establecido por Lastra y Martín Butragueño (2000).

De cada entrevista se seleccionaron 30 enunciados aseverativos o representativos (Félix-Brasdefer, 2016; Searle, 1976), de los cuales la mitad contienen al menos una frase entonativa intermedia (ip) y la otra mitad consiste únicamente en una frase entonativa mayor (IP). El total de 360 enunciados fue procesado a través del programa Praat 6.1.06 (Boersma y Weenink, 2019), con el cual se realizó la segmentación silábica de los enunciados, se extrajeron las medidas de duración de las sílabas que componen los tonemas de ip e IP (última acentuada y final) y se ejecutó la asignación de acentos tonales y tonos de juntura.

El sistema de notación prosódica empleado fue, principalmente, el modelo propuesto por Estebas-Vilaplana y Prieto (2008) con algunas adaptaciones tomadas de Frota y Prieto (2015) y Martín Butragueño y Mendoza (2018), todo ello reflejado en el apéndice 1. La asignación de acentos tonales y junturas se llevó a cabo en dos partes. Durante la primera fase se tomaron en cuenta las características fonéticas de la curva melódica en la que se usaron etiquetas como las de elevaciones tonales mayores a 3 semitonos $\left(\mathrm{L}+{ }_{¡} \mathrm{H}^{*}\right)$ (Martín Butragueño y Mendoza, 2018), la de pico entonativo muy temprano $\left(\mathrm{L}+<\mathrm{H}^{*}\right.$ ) (Martín Butragueño y Mendoza, 2018) y junturas bitonales (Frota y Prieto, 2015; Martín Butragueño y Mendoza, 2018; Estebas-Vilaplana y Prieto, 2008). Esto originó un número de configuraciones tonemáticas bastante elevado (48 configuraciones tonales finales de ip y 27 tonemas de IP), lo que hacía que el volumen de configuraciones fuese poco viable para su procesamiento. Por lo tanto, en una segunda fase, se sometieron las configuraciones tonales originales a un proceso de generalización de rasgos que prioriza las cualidades de los tonemas, por lo que se agruparon las diferentes etiquetas de elevación tonal $\left(\mathrm{L}+{ }_{j} \mathrm{H}^{*}, \mathrm{~L}+<\mathrm{H}^{*}, \mathrm{~L}+<_{i} \mathrm{H}\right)$ en 
una sola etiqueta que engloba todas estas posibilidades a la vez $\left(\mathrm{L}+\mathrm{H}^{\star}\right)$, al igual que, en el caso de las junturas bitonales, se tomó únicamente la altura del último tono de la secuencia (por ejemplo, HL\% y L\% se representan como L\%) debido a que este reproduce el valor pragmático que el hablante desea otorgarle a su ip o IP. Si bien es cierto que esta decisión puede afectar el grado de detalle del análisis entonativo, se considera que este proceso no perjudica el valor fonológico del análisis de las configuraciones tonales en los tonemas estudiados, pues se sigue apegando a los principios fonológicos del análisis métrico autosegmental explicado y aplicado por Pierrehumbert (1980) y Sosa (1999).

\section{Resultados}

Frases entonativas intermedias (ip): configuraciones tonales finales de ip

Debido a que entre las 267 ip extraídas del corpus se encuentran 74 que concluyen con acento oxítono, los análisis relativos a las configuraciones tonales finales de ip se dividen en dos grupos: configuraciones no oxítonas y configuraciones oxítonas. En cuanto al primero de estos grupos, tal como se observa en el gráfico 1, en cada red predominan las configuraciones tonales finales $\mathrm{H}^{*} \mathrm{H}-\mathrm{y} \mathrm{L}+\mathrm{H}^{*} \mathrm{H}$-, la primera de las cuales es privilegiada por la red laboral (con 19 casos de diferencia), mientras que la segunda es más frecuente en la red vecinal (aunque por solo 6 ocurrencias). El resto de las configuraciones son considerablemente menos frecuentes que las ya mencionadas, pues ninguna de ellas supera las 10 ocurrencias. Por ende, se puede afirmar que la tendencia mayoritaria de ambas redes consiste en una preferencia a mantener un tono elevado desde la sílaba nuclear de ip. Adicionalmente, la mayor frecuencia de la configuración $\mathrm{H}^{\star} \mathrm{H}$ - en las producciones de la red laboral demuestra su asentamiento como configuración nuclear de ip en dicha red, especialmente en contraste con lo que se observa en los casos de la red vecinal, cuya cercana frecuencia de uso de las configuraciones $\mathrm{H}^{\star} \mathrm{H}$ - y $\mathrm{L}+\mathrm{H}^{*} \mathrm{H}$ - no permite plantear que alguna de 
ellas esté más asentada que la otra o que pueda representar un rasgo identitario de esta red. Se puede observar un ejemplo de la configuración tonal final de ip $\mathrm{H}^{\star} \mathrm{H}$ - en la figura 1 y uno de la $\mathrm{L}+\mathrm{H}^{*} \mathrm{H}$ - en la figura 2 .

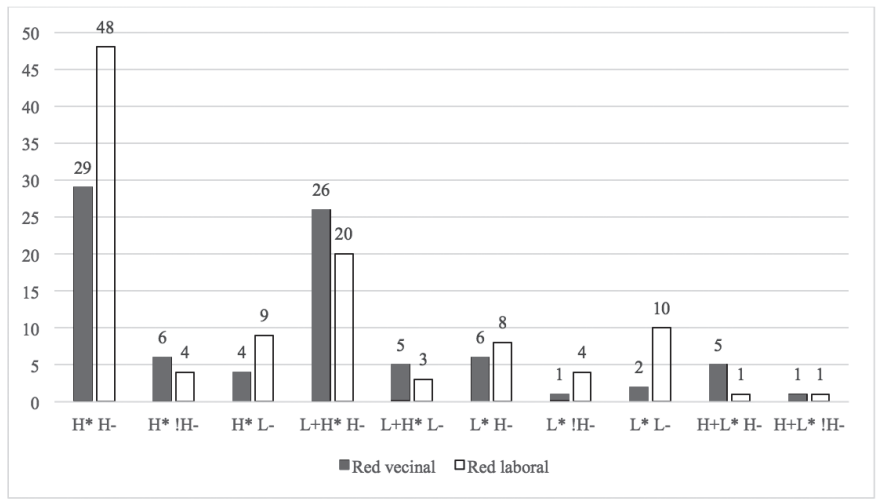

Gráfico 1. Recuento de casos de configuraciones tonales finales de ip no oxítonas en cada red

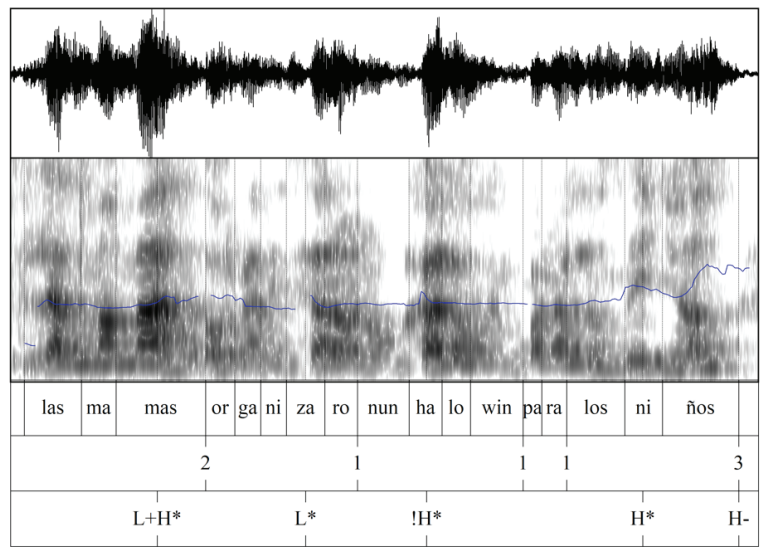

Figura 1. Ejemplo de configuración tonal final de ip $\mathrm{H}^{*} \mathrm{H}$ - en el enunciado

Rs2M1-13: [(Las mamás organizaron un Halloween para los niños)ip (pero lo hicieron el lunes a las dos de la tarde) ip]IP

La preferencia general por junturas altas concuerda con la elevación tonal al final de frase entonativa que caracteriza la continuidad o no conclusión del enunciado registrada en el español desde los trabajos clásico de Navarro Tomás (1957 y 1966) 


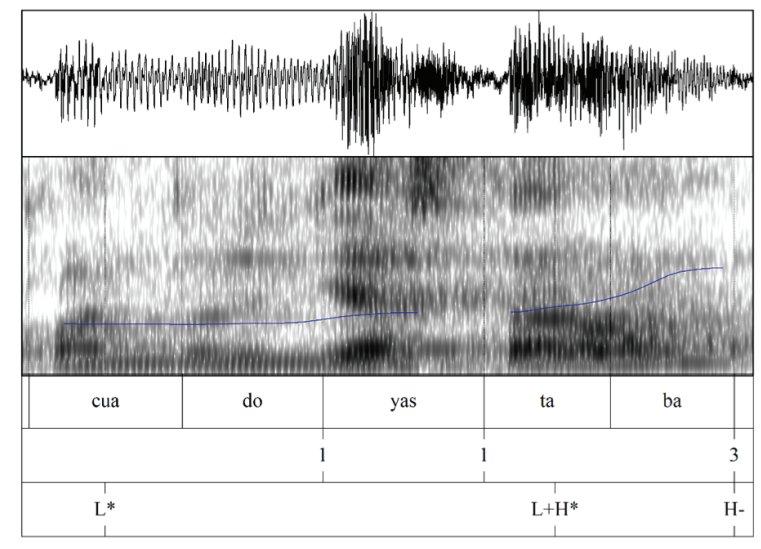

Figura 2. Ejemplo de la configuración tonal final de ip $\mathrm{L}+\mathrm{H}^{\star} \mathrm{H}$ - en el enunciado Rs1H3-21: [(Cuando ya estaba)ip (agarrabas un bote lo llenabas)ip (y se hacían unas planchas así como la mesa)ip]IP

y Quilis (1981), junto con las afirmaciones de Gussenhoven (2004) y Toledo (2007). Por tal motivo, tampoco sorprende la baja productividad de junturas L- en ip (como el mostrado en la figura 3), aunque resulta interesante que la red social laboral produzca la mayoría de los casos de esta juntura, especialmente en aquellas configuraciones tonales finales con acentos tonales mononucleares ( $\mathrm{L}^{\star} \mathrm{L}-\mathrm{y} \mathrm{H} \mathrm{H}^{\star} \mathrm{L}-$ ). Entre estas configuraciones, $\mathrm{L}^{*}$ L- es favorecida por la red laboral con una diferencia de 8 casos (la segunda desigualdad más abrupta entre ambas redes, superada únicamente por la de 19 casos registrada en la configuración $\mathrm{H}^{\star} \mathrm{H}$-). Dicho favorecimiento del uso de las junturas L- en configuraciones tonales con núcleos monotonales $\left(\mathrm{H}^{*} \mathrm{~L}-\mathrm{y} \mathrm{H} \mathrm{H}^{*} \mathrm{~L}-\right.$ ) por parte de la red laboral, especialmente notorio en las producciones de $\mathrm{L}^{*} \mathrm{~L}$-, apunta hacia una tendencia secundaria por parte de la red laboral a concluir las ip con entonación descendente. Dado que la frecuencia de uso es baja, esto no quiere decir que estas junturas estén particularmente asentadas en este grupo de hablantes, sino que puede representar un rasgo prosódico distintivo de esta red, teniendo en cuenta el contraste con la menos cantidad de casos generados por la red vecinal.

De estos 193 casos de configuraciones no oxítonas, predominan aquellas dadas en palabras paroxítonas (llanas) y úni- 


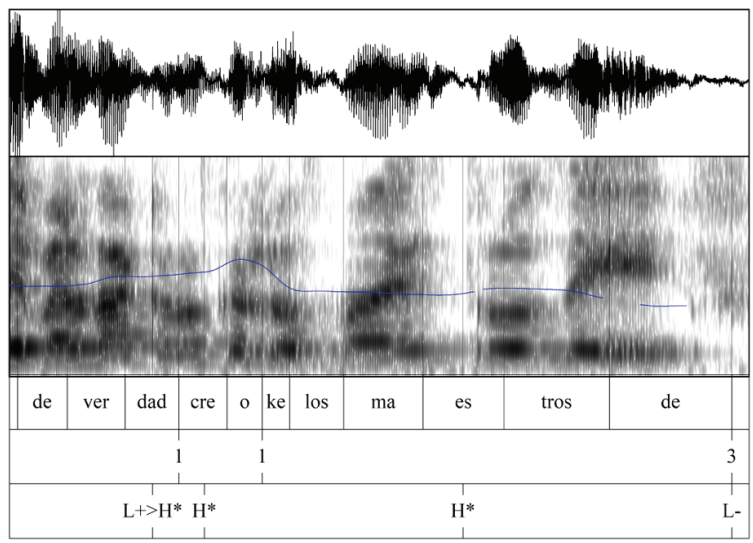

Figura 3. Ejemplo de configuración tonal final de ip $\mathrm{H}^{\star} \mathrm{L}$ - en el enunciado Rs2M2-26: [(De verdad creo que)ip (los maestros de primaria y de kinder)ip (tienen un reto bien fuerte)ip]IP

camente 18 se dan en palabras proparoxítonas (esdrújulas). La mayoría de estos 18 casos $(66.7 \%)$ posee núcleos monotonales. Los acentos nucleares altos $\left(\mathrm{H}^{*}\right)$ son seguidos tanto por junturas altas (H-) como bajas (L-) en igualdad de proporción, mientras que en los acentos bajos $\left(\mathrm{L}^{*}\right)$ la curva melódica únicamente desciende hasta L-, que es la juntura más común en las configuraciones tonales finales proparoxítonas. Aunado a esto, se registró una ausencia absoluta de configuraciones no finales con junturas medias (!H-), lo que da a pensar que la sílaba ubicada entre la nuclear y la final (la penúltima sílaba de la ip) brinda el tiempo necesario para que el tono llegue hasta la juntura H- o L-. No obstante, el limitado número de casos proparoxítonos hace imposible desde un punto de vista analítico poder hacer estas afirmaciones de forma categórica.

Con respecto a las configuraciones tonales finales de ip en palabras oxítonas al final de ip, los datos reflejan, como se aprecia en el gráfico 2, una tendencia general a la realización de elevaciones tonales $\left(\mathrm{L}+\mathrm{H}^{*}\right)$ a lo largo de la última sílaba que culminan en una juntura alta (H-). Solo 3 de todos los casos que presentan dicha elevación tonal culminan con juntura media (!H-), lo que demuestra la agudeza de la anticadencia en esta sí- 
laba final de ip. Una vez más, la poca preferencia de ambas redes por culminar las ip con tonos bajos se refleja en los 5 casos que presentan junturas L-. En términos de frecuencia de uso, ambas redes reflejan un comportamiento bastante uniforme, con pocos casos de diferencia entre una y otra en cada configuración tonal, por lo que sería arriesgado realizar afirmaciones categóricas en cuanto a la asociación de alguna configuración con cualquiera de las redes.

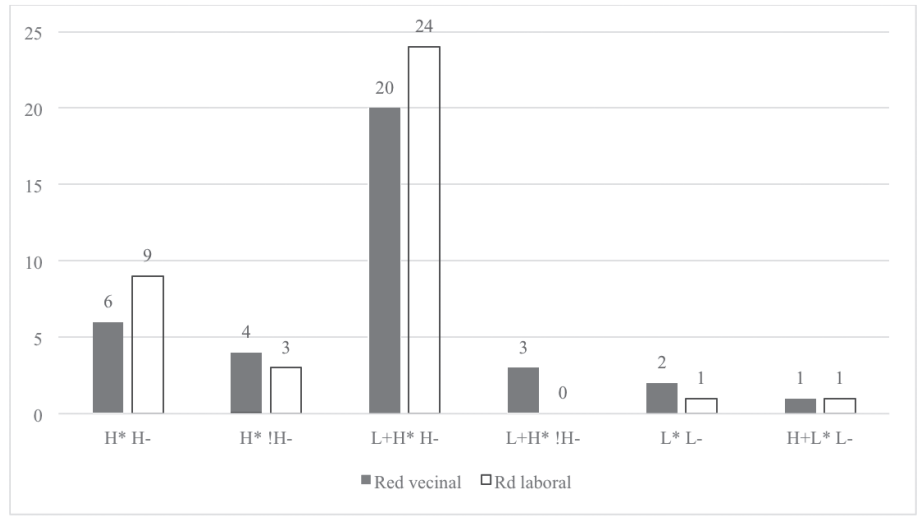

Gráfico 2. Recuento de casos de configuraciones tonales finales de ip oxítonas en cada red

Los datos del gráfico permiten tener una perspectiva del comportamiento de la curva melódica en la sección final de las ip en cada una de las redes sociales, pero para complementar este análisis se procederá a incluir la duración silábica en la ecuación. En términos de la media de la duración de las configuraciones tonales finales no oxítonas, se registró que ambas redes sociales producen sílabas nucleares más cortas que la sílaba linde de la ip (con una diferencia de $62.15 \mathrm{~ms}$ para la red vecinal y una de $49.17 \mathrm{~ms}$ para la red laboral). A pesar de que este comportamiento es bastante uniforme, en lo concerniente a la duración media de la sílaba nuclear se observa que la red laboral emite estas sílabas con una duración más elevada en la mayoría de las configuraciones. Una tendencia parecida no es observable en la duración de las sílabas lindes de ip, pues las medias de duración carecen de uniformidad en relación con las otras dos 
variables (configuraciones tonales finales de ip y redes sociales), por lo que no se puede observar un patrón que permita relacionar la duración de la sílaba nuclear o linde en las producciones de ambas redes.

El caso de las configuraciones tonales finales oxítonas es muy diferente al de las no oxítonas. Como se puede observar en el cuadro 1, la media de duración de las últimas sílabas acentuadas de las ip para todos los tonemas es mucho más elevada en la red social laboral que en la vecinal (teniendo en cuenta que la configuración tonal final $\mathrm{L}+\mathrm{H}^{*} ! \mathrm{H}-$ no presenta casos en la red laboral). Esta inclinación que presenta la red laboral a producir la sílaba final tónica con duración media mayor a los 300 ms se ve reafirmada por la frecuencia con la que esto ocurre. De las 38 sílabas portadoras de configuraciones finales oxítonas de ip documentadas en esta red, 20 de ellas (52.6\%) superan los 300 ms de duración, mientras que de los 36 casos registrados en la red vecinal, solo 9 de ellos (25\%) presentan esta tendencia. Esto apunta hacia una marcada diferencia en el grado de asentamiento de esta característica prosódica en ambas redes. Para ilustrar este alargamiento, en la figura 4 se muestra un ejemplo del comportamiento descrito para la red laboral, en donde se evidencia la prologada duración de la sílaba final (con acento oxítono).

\begin{tabular}{|c|c|c|}
\hline Configuraciones tonales oxítonas & Red vecinal & Red laboral \\
\hline $\mathrm{H}^{*} \mathrm{H}-$ & 253.33 & 303.56 \\
\hline $\mathrm{H}^{*} ! \mathrm{H}-$ & 277.5 & 306.67 \\
\hline $\mathrm{L}+\mathrm{H}^{*} \mathrm{H}-$ & 243.65 & 302.42 \\
\hline $\mathrm{L}+\mathrm{H}^{*} ! \mathrm{H}-$ & 161 & 0 \\
\hline $\mathrm{L}^{*} \mathrm{~L}-$ & 164 & 392 \\
\hline $\mathrm{H}+\mathrm{L}^{*} \mathrm{~L}-$ & 288 & 336 \\
\hline Total & 238.94 & 306.26 \\
\hline
\end{tabular}

Cuadro 1. Media de duración de configuraciones tonales finales de ip oxítonas en cada red

Ahora bien, el análisis de las medias permite tener una perspectiva clara acerca de las dimensiones del correlato acústico de 


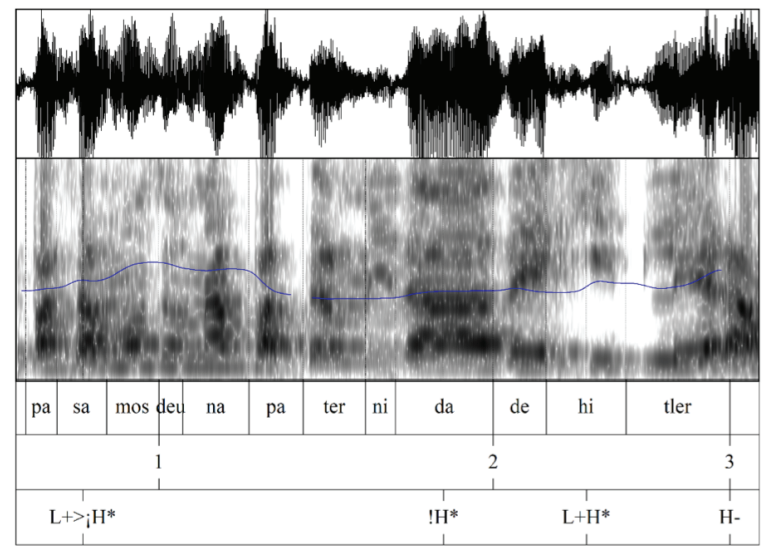

Figura 4. Ejemplo de configuración tonal final de ip $\mathrm{H}^{*} \mathrm{~L}$ - en el enunciado Rs2M2-26: [(Pasamos de una paternidad de Hitler)ip (a una paternidad completamente permisiva)ip ]IP

la duración en las sílabas que involucran la configuración tonal final de ip, pero estas medias no permiten observar lo que ocurre en términos de uso. Para ello es necesario prestar atención a lo que ocurre en términos de la duración relativa de las sílabas finales de ip. Al realizar dicho análisis en las configuraciones tonales finales de ip, donde se considera únicamente si la duración de la sílaba nuclear es mayor o menor que la de la sílaba linde de ip, se observa que la frecuencia con la que ambas redes sociales producen sílabas finales más largas que las nucleares es más del doble de los casos en los que ocurre lo contrario. Otro hecho general que llama la atención es que las configuraciones tonales que involucran el acento nuclear $\mathrm{H}+\mathrm{L}^{*}$ son siempre producidas con sílabas nucleares más cortas que las del linde de ip por ambas redes sociales. No se puede decir lo mismo de las sílabas nucleares con acentos bitonales ascendentes $\left(\mathrm{L}+\mathrm{H}^{\star}\right)$, pues aunque su duración es mayoritariamente más corta que las del linde de ip, sí se registran casos de lo inverso. Por ende, no se puede atribuir la ausencia absoluta de sílabas nucleares más largas a la presencia de acentos bitonales dado que esta restricción solo es visible en aquellos que son descendentes $\left(\mathrm{H}+\mathrm{L}^{*}\right)$, independientemente del tono de juntura que les siga. Por otra parte, la duración relativa de ambas sílabas de configuración tonal final de 
ip no aporta indicios acerca de una posible tendencia específica por parte de alguna de las redes, ya que las frecuencias de las ocurrencias registradas son bastante uniformes y las diferencias identificadas entre una y otra red son muy pequeñas como para aventurar la propuesta de algún patrón de conducta prosódica relacionado con las características sociales de cualquiera de las redes.

Frases entonativas mayores (IP): tonemas

Los 180 tonemas registrados por cada red social, todos ellos dados en palabras paroxítonas, dan cuenta del predominio de los tonos de juntura bajos (L\%) sobre todos los demás, dado que esta se observa en el $87.78 \%$ de todos los tonemas del corpus. Esto no resulta sorprendente debido a que las junturas L\% en enunciados aseverativos ya han sido documentadas como un hecho común para varios dialectos del español (Sosa, 1999). La frecuencia de ocurrencia de los tonemas que culminan con junturas $\mathrm{L} \%$, en orden decreciente, es el siguiente: $\mathrm{L}^{\star} \mathrm{L} \%(33.33 \%), \mathrm{H}^{\star}$ $\mathrm{L} \%$ (27.5\%), $\mathrm{L}+\mathrm{H}^{\star} \mathrm{L} \%$ (18.89\%) y $\mathrm{H}+\mathrm{L}^{\star} \mathrm{L} \%$ (8.06\%). La mayor frecuencia del tonema $\mathrm{L}^{\star} \mathrm{L} \%$ concuerda con los movimientos tonales de la declinación melódica natural que se da al final del enunciado (Gussenhoven, 2004) y que se ha establecido como una característica del español (D’Introno et al., 2010; Navarro Tomás, 1957 y 1966). De igual modo, se registra un porcentaje importante del tonema circunflejo $\mathrm{L}+\mathrm{H}^{*} \mathrm{~L} \%$ (visible en la figura 5), característico del habla del centro de México (Kvavik, 1979; Martín Butragueño, 2006; Sosa, 1999).

La distribución de casos de los tonemas por red, mostrados en el gráfico 3, permiten observar que, si bien los tonemas con junturas L\% son los más comunes en ambas redes, la red laboral los produce con más frecuencia en todas las instancias, con excepción del tonema circunflejo, más favorecido por la red vecinal. Curiosamente, esta misma red presenta mayor frecuencia de los tonemas $\mathrm{L}+\mathrm{H}^{*} \mathrm{H} \%$ y $\mathrm{L}+\mathrm{H}^{*} ! \mathrm{H} \%$, lo que refleja una tendencia secundaria, mucho menos frecuente, por los hablantes de esta red. 


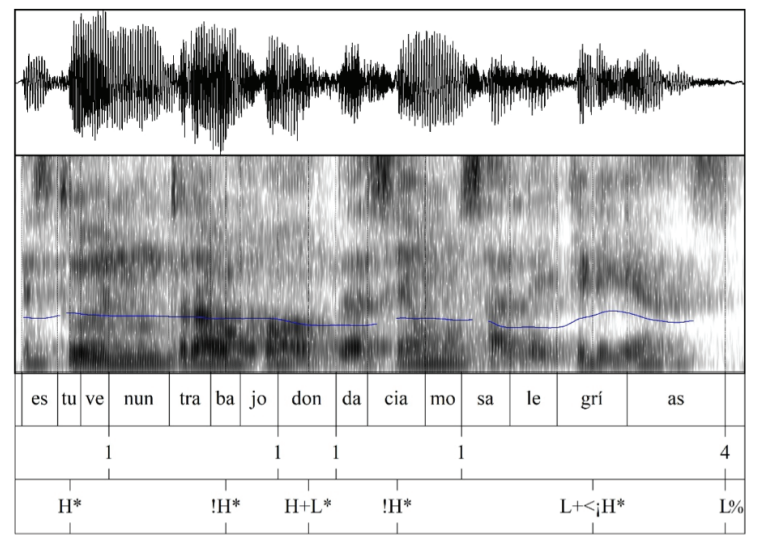

Figura 5. Ejemplo de tonema $\mathrm{L}+\mathrm{H}^{\star} \mathrm{L} \%$ en el enunciado Rs1H3-19: [Estuve en un trabajo donde hacíamos alegrías]IP

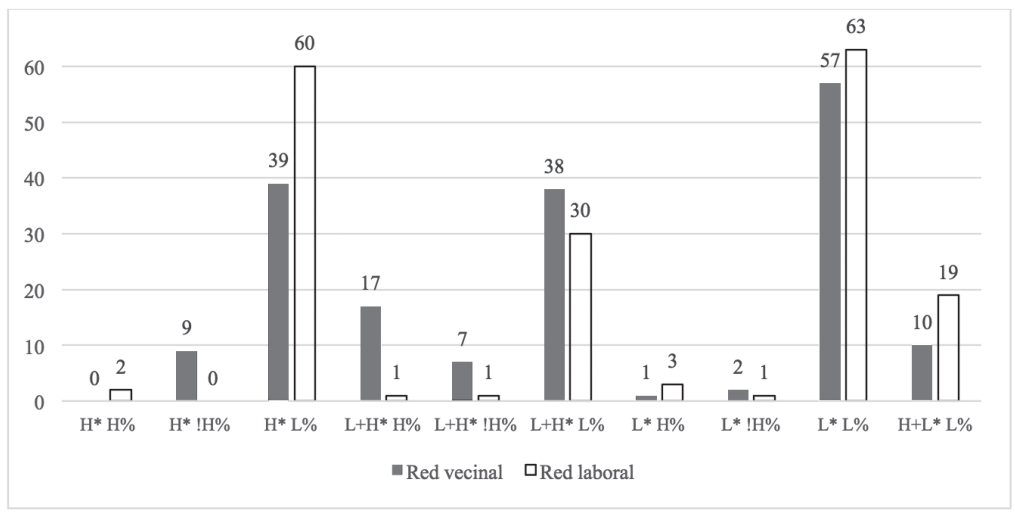

Gráfico 3. Recuento de casos de tonemas en cada red

Con respecto a la duración media de las sílabas que integran el tonema, se observó que las medias de duración tanto de sílaba nuclear como de sílaba linde son mayores en la red vecinal que en la laboral, lo que señala una tendencia por parte de la primera red a elongar las sílabas finales de las IP. Otra diferencia llamativa es que las redes sociales estudiadas presentan comportamientos opuestos en cuanto a la sílaba con mayor prominencia cronológica: mientras la media de duración de la sílaba final (192.02 ms) es mayor que la del núcleo $(183.93 \mathrm{~ms})$ en la red 
vecinal, la red laboral produce sílabas nucleares más largas que las finales (172.23 ms y $157.25 \mathrm{~ms}$, respectivamente).

En términos de duración relativa, no hay una orientación común en las dos redes con relación a la frecuencia con la que se alarga la sílaba nuclear en detrimento de la del linde y viceversa. Por una parte, la red vecinal registra la misma frecuencia de prolongaciones de las sílabas nucleares que de contracciones de esta (90 en cada caso). La diferencia entre la elongación de una sílaba con respecto a la otra es bastante reducida en todos los tonemas (no supera los 5 casos de diferencia); no obstante, dos tonemas presentan una diferencia mayor: $\mathrm{H}^{\star} \mathrm{L} \%$ y $\mathrm{L}^{\star} \mathrm{L} \%$. Esta red social tiende a producir el primero principalmente con sílabas nucleares más largas que la final, mientras que hacen lo contrario con respecto al segundo tonema. Por lo tanto, teniendo en cuenta la frecuencia de los casos, es posible señalar que la red vecinal no se identifica con la mayor prolongación de alguna de las dos sílabas finales IP en concreto, excepto en los casos de los tonemas $\mathrm{H}^{*} \mathrm{~L}-\mathrm{y} \mathrm{L}^{\star} \mathrm{L}$-. Por otra parte, la red laboral refleja mayor frecuencia de sílabas nucleares más largas que las finales, pues 115 de las ocurrencias corresponden con este planteamiento, mientras que 65 se dan de forma contraria. En todos los tonemas, esta red prolonga la sílaba nuclear en detrimento de la final, excepto en el $\mathrm{L}^{*} \mathrm{H} \%$, aunque la diferencia a favor de este tonema es bastante reducida (solo un caso). De hecho, la diferencia de duración relativa en la mayoría de los demás tonemas es igualmente reducida, con una diferencia no mayor a 6 casos, con excepción del tonema circunflejo $\mathrm{L}+\mathrm{H}^{*} \mathrm{~L} \%$ (12 casos de diferencia) y $\mathrm{H}+\mathrm{L}^{*} \mathrm{~L} \%$ y $\mathrm{L}^{\star} \mathrm{L} \%$ (cada uno con $15 \mathrm{ocu}-$ rrencias de diferencia a favor de sílabas nucleares más largas). Todo lo dicho en el caso de la red laboral evidencia el hecho de que el uso de sílabas nucleares más largas que las finales es un rasgo característico de esta red, especialmente teniendo en cuenta lo ya mencionado acerca de los casos de duración relativa en la red vecinal.

\section{Conclusiones}

En relación con las configuraciones tonales finales de ip no oxítonas, se observa que la tendencia mayoritaria es el uso de anti- 
cadencias originadas desde la sílaba nuclear, de entre las cuales $\mathrm{H}^{*} \mathrm{H}$ - es más empleada por la red laboral y $\mathrm{L}+\mathrm{H}^{*} \mathrm{H}$ - por la vecinal. Dicha elevación tonal es un recurso empleado por los hablantes para transmitir un sentido de continuidad o no conclusión de la enunciación (Gussenhoven, 2004) que ha sido registrado continuamente para el español (Navarro Tomás, 1957 y 1966; Quilis, 1981; Toledo, 2007). No obstante, el hecho de que $17.1 \%$ de las configuraciones presenten junturas L- hace pensar acerca de la necesidad de un estudio posterior relacionado con las características pragmáticas de dichas junturas bajas.

Entre las configuraciones tonales finales de ip proparoxítonas, llamó la atención la ausencia de junturas medias (!H-) y que los acentos tonales $\mathrm{L}^{\star}$ siempre son seguidos por junturas L-. Esto no necesariamente es señal de la existencia de una restricción en estos casos, pues no se puede descartar la posibilidad de que no se hayan registrado otras posibilidades debido al reducido número de casos estudiados o por otros elementos pragmáticos que no se hayan tomado en consideración en esta investigación. Para poder establecer una relación categórica entre las configuraciones proparoxítonas y el predominio de la juntura L- junto con la ausencia de junturas medias (!H-), sería necesario hacer un estudio donde se controlen las variables pragmáticas que pudieran afectar los contornos melódicos de estos casos en concreto.

La sílaba final de ip es relevante en términos cronológicos no solo por su mayor duración media, sino también por la frecuencia con la que ésta es prolongada en detrimento de la sílaba nuclear. Esto se debe a que ambas redes sociales producen sílabas finales de ip con una duración media más prolongada que la de la sílaba nuclear de ip. Este alargamiento en el linde derecho de las frases entonativas intermedias (ip) también se da con una frecuencia mayor al doble de los casos en los que ocurre lo contrario, lo que evidencia su relevancia en el uso lingüístico de los hablantes.

Las configuraciones tonales finales de ip oxítonas presentan un patrón similar al de las no oxítonas: favorecimiento general de elevaciones tonales $\left(\mathrm{L}+\mathrm{H}^{*}\right)$ que finalizan con junturas altas (H-). La duración de esta sílaba tónica final de ip represen- 
ta una diferencia categórica entre ambas redes. La red laboral presenta medias de duración en esta sílaba final que son considerablemente mayores que las reflejadas por la red vecinal, con una diferencia promedio de $42.2 \mathrm{~ms}$. Esto, conjugado con el elevado uso de una duración superior a los 300 ms en estas sílabas por parte de la red laboral y con la mayor frecuencia con la que estas sílabas son producidas con configuraciones tonales en la parte alta del campo tonal $\left(\mathrm{H}^{*} \mathrm{H}-\mathrm{y} \mathrm{L}+\mathrm{H}^{*} \mathrm{H}-\right)$, apunta hacia el siguiente patrón prosódico característico de esta red: producción de configuraciones tonales finales de ip oxítonas con una prolongada emisión de un acento tonal alto $\left(\mathrm{H}^{\star}\right)$ o ascendente $\left(\mathrm{L}+\mathrm{H}^{*}\right)$ que culmina en el rango tonal superior (juntura $\mathrm{H}_{-}$) de los hablantes.

La sistematicidad observada en las configuraciones tonales finales de ip (especialmente marcada en la duración que cada red presenta en los casos de los oxítonos) da pie a pensar en la pertinencia y relevancia del planteamiento de la frase entonativa intermedia (ip) como un nivel relevante de la jerarquía prosódica del español. Se observan patrones conductuales recurrentes que relacionan fonológicamente el significado dependiente de cada ip dentro de una unidad de significado mayor e independiente (la IP) por medio de recursos prosódicos que se manifiestan a través de tendencias globales de la comunidad de habla y que, al mismo tiempo, pueden presentar rasgos particulares en determinados grupos de habla.

Por otra parte, los tonemas de IP presentan patrones melódicos bastante diferentes a los observados en las ip. En primer lugar, se registra un predominio de junturas L\% (87.78\% del total), entre los que destaca $L^{\star} L \%$ por ser el más favorecido por ambas redes (33.3\% de los casos). El uso del tonema circunflejo $\mathrm{L}+\mathrm{H}^{*} \mathrm{~L} \%$ (el tercero más frecuente en el corpus, presente en el $18.89 \%$ de los enunciados analizados) es preferido, aunque no en gran medida, por los hablantes de la red social vecinal, por lo que no se puede afirmar categóricamente que sea un rasgo característico de esta red. Cabe señalar que los miembros de la red vecinal también favorecen el uso de junturas medias (!H\%) $\mathrm{y}$ altas $(\mathrm{H} \%)$ en IP, especialmente cuando el acento nuclear es ascendente $\left(\mathrm{L}+\mathrm{H}^{*}\right)$. Este comportamiento poco esperable, que 
podría ser considerado como una tendencia secundaria por parte de la red vecinal debido a la diferencia que presenta con respecto a la red laboral, merece ser estudiada a mayor profundidad en combinación con otras variables (como expresividad, focalización, entre otras que se puedan considerar pertinentes) de forma tal que se pueda llegar a generar una explicación que permita identificar los factores que puedan estar asociados a este comportamiento lingüístico poco habitual (aunque no extraño $y$, definitivamente, no imposible).

Finalmente, con respecto a la duración silábica en el tonema, no se observa una tendencia general en términos de duración absoluta ni relativa. Por una parte, la red vecinal produce tonemas más largos que los de la otra red al prolongar más las sílabas finales de IP, entre las cuales la sílaba final es, es promedio, más larga que la nuclear. No obstante, dicho alargamiento de la sílaba final no se refleja en la duración relativa, pues esta red produce sílabas nucleares más largas que las finales con la misma frecuencia con la que hace lo opuesto. Por ende, la única conclusión alcanzable es que, cuando la sílaba final del enunciado es más larga que la nuclear, su duración media es mayor que la de las sílabas nucleares en aquellos casos en los que estas son más largas que la final. Por otra parte, los tonemas producidos por la red social laboral siguen un patrón más claro, pues la duración media de las sílabas nucleares no solo es más larga que las finales, sino que la frecuencia con la que son más largas que la sílaba final también es mayor, especialmente en los tonemas que implican un descenso tonal hasta una juntura $\mathrm{L} \%$ $\left(\mathrm{L}+\mathrm{H}^{\star} \mathrm{L} \%, \mathrm{H}+\mathrm{L}^{\star} \mathrm{L} \%\right.$ y $\left.\mathrm{L}^{\star} \mathrm{L} \%\right)$.

\section{Referencias}

Boersma, P. y Weenink D. (2019). Praat: Doing Phonetics by Computer [Programa informático]. Versión 6.1.06. Recuperado de http://www.fon.hum.uva.nl/praat/.

Bybee, J. (2001). Phonology and Language Use. Cambridge: Cambridge University Press.

D’Introno, F., Del Teso, E. y Weston, R. (2010). Fonética y fonología del español. Madrid: Cátedra. 
Estebas-Vilaplana, E. y Prieto, P. (2008). La notación prosódica del español: una revisión del Sp_ToBI. Estudios de Fonética Experimental, 27, 263-283.

FÉlix-Brasdefer, J. C. (2016). Actos de habla. En J. GutiérrezRexach (ed.), Enciclopedia de lingüística hispánica, vol. 2 (pp. 201-212). Nueva York: Routledge.

Frota, S. y Prieto, P. (2015). Intonation in Romance. Oxford: Oxford University Press.

Gussenhoven, C. (2004). The Phonology of Tone and Intonation. Nueva York: Cambridge University Press.

Heusinger, K. von (2007). Discourse Structure and Intonational Phrasing. En L. Chungmin, M. Gordon y D. Büring (eds.), Topic and Focus: Cross-Linguistic Perspectives on Meaning and Intonation (pp. 265-290). Dordrecht: Springer.

Hualde, J. I. (2003). El modelo métrico autosegmental. En P. Prieto (ed.), Teorías de la entonación (pp. 155-184). Barcelona: Ariel.

KvaviK, K. (1979). An Interpretation of Cadences in Mexican Spanish. En J. P. Lantolf, F. Wattman Frank y J. M. Guitart (eds.), Colloquium on Spanish and Luso-Brazilian Linguistics (pp. 34-74). Washington: Georgetown University Press. Labov, W. (1981). Field Methods of the Project on Linguistic Change and Variation, Sociolinguistic Working Paper Number 81. Austin: Southwest Educational Development.

Lastra, Y. y Martín Butragueño, P. (2000). El modo de vida como factor sociolingüístico en la Ciudad de México. En P. Martín Butragueño (ed.), Estructuras en contexto: Estudios de variación y cambio (pp. 13-43). México: El Colegio de México.

Martín Butragueño, P. (2006). El estudio de la entonación en el español de México. En M. Sedano, A. Bolívar y M. Shiro (comps.), Haciendo lingüística: homenaje a Paola Bentivoglio (pp. 105-125). Caracas: Universidad Central de Venezuela. Martín Butragueño, P. (2015). Hacia una prosodia basada en el uso: actos de habla en el español mexicano. Revista Normas, 5, 97-115.

Martín Butragueño, P. (2019). Fonología variable del español de México, vol. II: Prosodia enunciativa, tomo 1. México: El Colegio de México. 
Martín Butragueño, P. y Mendoza, E. (2018). Prosodic Nuclear Patterns in Narrow and Broad Focus Utterances: Pragmatic and Social Factors in Central Mexican Spanish. En M. García García y M. Uth (eds.), Focus Realization in Romance and Beyond (pp. 131-172). Ámsterdam: John Benjamins.

MATluck, J. (1952). La pronunciación del español en el valle de México. Nueva Revista de Filología Hispánica, 6, 109-120.

Navarro Tomás, T. (1957). Manual de pronunciación española. Nueva York: Hafner Publishing.

Navarro Tomás, T. (1966). Manual de entonación española (3a ed.). México: Colección Málaga.

Nespor, M. y Vogel, I. (2007). Prosodic Phonology: with a new Foreword. Berlín: Mouton de Gruyter.

Pierrehumbert, J. (1980). The Phonology and Phonetics of English Intonation (tesis inédita de doctorado). Massachusetts Institute of Technology, Cambridge, Estados Unidos de América.

Prieto, P. (2006). Phonological Phrasing in Spanish. En S. Colina y F. Martínez-Gil (eds.), Optimality-Theoretic Advances in Spanish Phonology (pp. 60-69). Ámsterdam, John Benjamins.

Quilis, A. (1981). Fonética acústica de la lengua española. Madrid: Gredos.

SeArle, J. (1976). A Classification of Illocutorionary Acts. Language in Society, 5( 1), 1-23.

Selkirk, E. (1986). On Derived Domains in Sentence Phonology. Phonology Yearbook, 3, 371-405.

SosA, J. M. (1999). La entonación del español: su estructura fónica, variabilidad y dialectología. Madrid: Cátedra.

Toledo, G. (2007). Fraseo en español peninsular y modelo autosegmental y métrico. Estudios Filológicos, 42, 227-243.

Toledo, G. (2008a). Frases fonológicas $(\phi)$. Ianua. Revista Philologica Romanica, 8, 1-18.

Toledo, G. (2008b). Fonología de la frase entonativa. Estudios Filológicos, 43, 207-222. 


\section{Apéndices}

\begin{tabular}{|c|c|c|}
\hline \multicolumn{3}{|l|}{ Acentos tonales monotonales } \\
\hline & $\mathrm{L}^{*}$ & $\begin{array}{l}\text { Acento fonéticamente reali- } \\
\text { zado como una meseta baja } \\
\text { situada en el límite inferior del } \\
\text { rango tonal del hablante. } \\
\text { (Estebas-Vilaplana y Prieto, } \\
2008 \text { ) }\end{array}$ \\
\hline & $\mathrm{H}^{*}, ! \mathrm{H}^{*}$ & $\begin{array}{l}\text { Este acento se visualiza como } \\
\text { una meseta alta que no es } \\
\text { precedida por un valle de F0. } \\
\text { Cuando la meseta no es tan } \\
\text { elevada, } \mathrm{H}^{*} \text { se representa con } \\
\text { la forma ! } \mathrm{H}^{*} \text {. } \\
\text { (Martín Butragueño y Men- } \\
\text { doza, 2018) }\end{array}$ \\
\hline \multicolumn{3}{|l|}{ Acentos tonales bitonales } \\
\hline & $\mathrm{L}+\mathrm{H}^{*}$ & $\begin{array}{l}\text { Este acento es realizado foné- } \\
\text { ticamente como un ascenso } \\
\text { tonal durante la sílaba acen- } \\
\text { tuada, en cuyo final se en- } \\
\text { cuentra el pico de F0. } \\
\text { (Estebas-Vilaplana y Prieto, } \\
2008 \text { ) }\end{array}$ \\
\hline & $\mathrm{L}^{*}+\mathrm{H}$ & $\begin{array}{l}\text { Este acento consiste en un va- } \\
\text { lle de F0 en la sílaba acentuada } \\
\text { con un posterior ascenso en la } \\
\text { sílaba postónica. } \\
\text { (Estebas-Vilaplana y Prieto, } \\
2008 \text { ) }\end{array}$ \\
\hline & $\mathrm{H}+\mathrm{L}^{*}$ & $\begin{array}{l}\text { Este acento se realiza fonéti- } \\
\text { camente como un descenso } \\
\text { de F0 dentro de la sílaba acen- } \\
\text { tuada. } \\
\text { (Estebas-Vilaplana y Prieto, } \\
2008 \text { ) }\end{array}$ \\
\hline
\end{tabular}




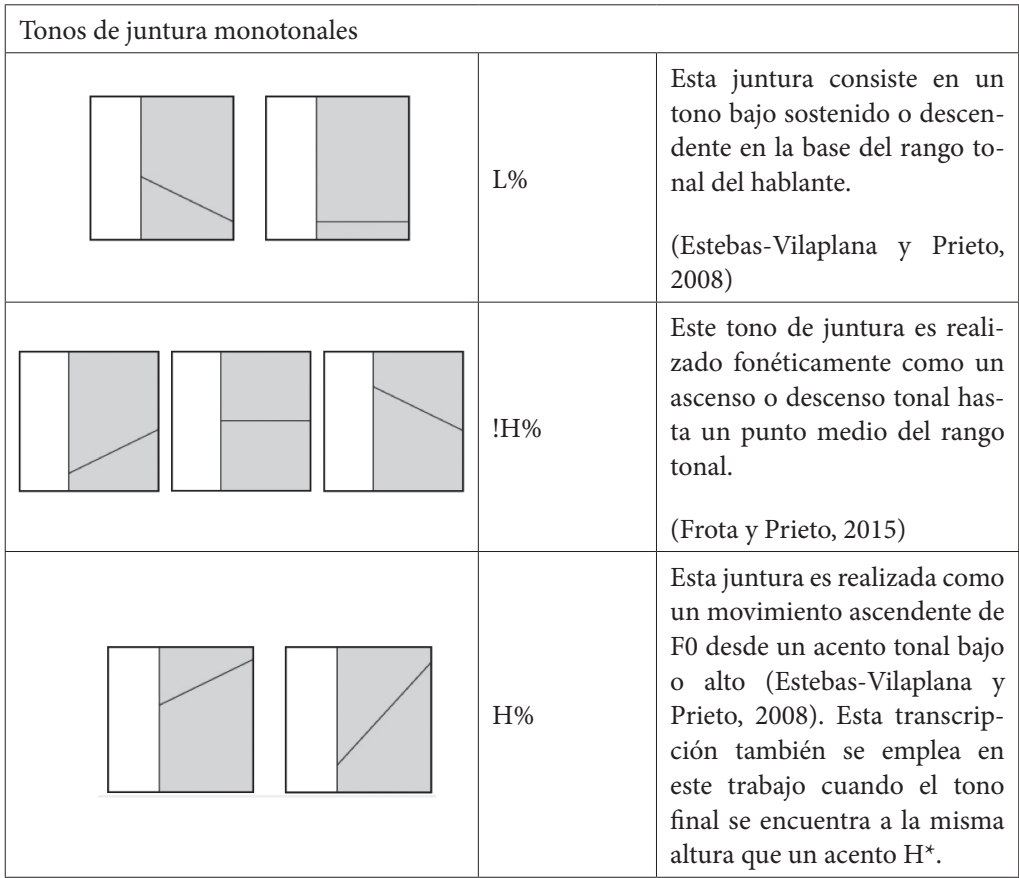

Apéndice 1. Gráfico ilustrativo y explicación del modelo del sistema de transcripción prosódica Sp_ToBI empleado en este trabajo 